\title{
Toracostomía abierta: en ocasiones la mejor opción
}

\author{
Open thoracostomy: Sometimes the best option
}

\author{
Camilo Ramírez-Giraldo ${ }^{1} \mathbb{D}$, Fiamma Vásquez ${ }^{2} \mathbb{D}$, Mauricio Peláez-Arango ${ }^{3} \mathbb{D}$
}

Médico, especialista en Epidemiología, residente de Cirugía General, Universidad del Rosario, Bogotá, D.C., Colombia.
Médica, residente de Cirugía General, Universidad del Rosario, Bogotá, D.C., Colombia.
Médico, especialista en Cirugía General, subespecialista en Cirugía de Tórax, Hospital Universitario Mayor, Bogotá, D.C., Colombia

\section{Resumen}

El tratamiento quirúrgico del empiema ha evolucionado, desde la medicina hipocrática, a través de los años, teniendo como premisa el drenaje. Con los avances médicos, el abordaje mínimamente invasivo se ha convertido en el estándar, dejando de lado otras técnicas quirúrgicas. La toracostomía abierta, en pacientes seleccionados, puede llegar a ser la última y mejor opción para el control del foco infeccioso: es una opción quirúrgica para el tratamiento de los empiemas en pacientes mórbidos y en fase de organización, cuando otros tratamientos han fallado, con una aceptable morbilidad y mortalidad. Esta técnica debe estar en el arsenal terapéutico del cirujano.

Presentamos una serie de 4 casos de pacientes con empiema en fase de organización, en los cuales el riesgo quirúrgico era muy alto, por lo que se optó por realizar una toracostomía abierta, con éxito.

Palabras clave: toracostomía abierta; tórax; empiema pleural; cirugía torácica; cirugía torácica asistida por video.

\begin{abstract}
The surgical treatment of empyema has evolved from Hippocratic medicine over the years, with drainage as its premise. With medical advances, the minimally invasive approach has become the standard, leaving aside other surgical techniques. Open thoracostomy, in selected patients, may become the last and best option for the control of the infectious focus: it is a surgical option for the treatment of empyemas in morbid patients and in the organization phase when other treatments have failed, with an acceptable morbidity and mortality. This technique should be in the surgeon's therapeutic arsenal. We present a series of four cases of patients with empyema in the organization phase, in which the surgical risk was very high, so it was decided to perform an open thoracostomy, which turn out successful.
\end{abstract}

Keywords: open thoracostomy; thorax; empyema, pleural; thoracic surgery; thoracic surgery, video-assisted.

Fecha de recibido: 01/05/2020 - Fecha de aceptación: 01/07/2020

Correspondencia: Camilo Ramírez Giraldo, Hacienda Fontanar del Río, Alcaparro 78, km 2 vía Chía - Cajicá, Chía, Colombia. Celular: 3206770474

Correo electrónico: ramirezgiraldocamilo@gmail.com

Citar como: Ramírez-Giraldo C, Vásquez F, Peláez-Arango M. Toracostomía abierta: en ocasiones la mejor opción. Rev Colomb Cir. 2021;36:60-5. https://doi.org/10.30944/20117582.638

Este es un artículo de acceso abierto bajo una Licencia Creative Commons - BY-NC-ND https://creativecommons.org/licenses/by-ncnd/4.0/deed.es 


\section{Introducción}

El empiema es una entidad prevalente, en la que se genera un proceso inflamatorio en el espacio pleural, con una sustancial morbimortalidad. Frecuentemente ocurre secundario a un proceso infeccioso subyacente a nivel pulmonar (neumonía, absceso pulmonar, bronquiectasias), pero también puede ser secundario a otras entidades, como procedimientos quirúrgicos, trauma con ruptura esofágica, o extensión de absceso hepático, entre otras.

Se caracteriza por tres fases, que se diferencian entre sí por el tiempo de evolución, las características del líquido pleural y la formación de fibrina con atrapamiento del pulmón afectado. Estas fases son: exudativa, que ocurre en los primeros días; fibrinopurulenta, que se presenta entre los siguientes 2 a 7 días, y una última fase de organización, que inicia a los 7 hasta 28 días y se caracteriza por su cronicidad ${ }^{1-3}$.

El tratamiento quirúrgico del empiema ha evolucionado a través de los años, desde la medicina hipocrática, teniendo como premisa el drenaje, como se expresa en la siguiente frase: "Ubi pus, ibi evacua" (donde hay pus, hay que evacuarlo) ${ }^{4}$. Con los avances médicos, el abordaje mínimamente invasivo se ha convertido en el estándar, dejando de lado otras técnicas quirúrgicas, como la toracostomía abierta, que es un procedimiento mutilante ${ }^{1,5}$.

Sin embargo, la toracostomía abierta en pacientes seleccionados puede llegar a ser la última y mejor opción para el control del foco infeccioso ${ }^{6}$. Entre las desventajas descritas se encuentran, la necesidad de la sección de músculos o costillas, que resulta en un defecto de la pared torácica, dolor crónico y afectación del aspecto cosmético. Adicionalmente, también se requiere atención especial de la herida con curaciones frecuentes para lograr la obliteración del espacio pleural, lo cual consume tiempo, es doloroso y el cierre de la pared no siempre es posible ${ }^{7,8}$. La terapia de presión negativa asociada a la toracostomía abierta, puede solucionar este problema, acelerando la obliteración del espacio pleural, con un recambio de curación menos frecuente, ayudando al cierre de las heridas más rápidamente, ofreciendo una mejor calidad de vida para los pacientes y una baja tasa de reinfección ${ }^{8,9}$.

\section{Métodos}

Estudio descriptivo que presenta una serie de 4 casos de pacientes que recibieron manejo con toracostomía abierta para el tratamiento de empiema crónico (fase de organización), en quienes habían fallado otras intervenciones quirúrgicas. Se hizo revisión retrospectiva de las historias clínicas e imágenes diagnósticas.

\section{Resultados}

En la tabla 1 se resumen las características clínicas de los cuatro pacientes a los que se les realizó toracostomía abierta.

\section{Caso clínico 1}

Paciente masculino de 61 años de edad, sin comorbilidades, con antecedente de neumonía y derrame paraneumónico, que recibió tratamiento antibiótico, drenaje y decorticación por VATS (cirugía torácica asistida por video), con posterior egreso por evolución clínica satisfactoria. Reconsulta a los 10 días por presentar secreción purulenta por herida quirúrgica y fiebre, y se le encuentra con respuesta inflamatoria sistémica dada por taquicardia, leucocitosis y PCR

Tabla 1. Características de los pacientes tratados con toracostomía abierta.

\begin{tabular}{cccccccc}
\hline Caso & Edad & Sexo & Recurrente & Fase & Tratamiento previo & ASA & Seguimiento \\
\hline 1 & 61 & $\mathrm{M}$ & $\mathrm{Si}$ & Crónica & VATS & III & 12 meses \\
2 & 60 & $\mathrm{~F}$ & $\mathrm{Si}$ & Crónica & VATS & III & 8 meses \\
3 & 51 & $\mathrm{M}$ & $\mathrm{No}$ & Crónica & Toracostomía cerrada & III & 18 meses \\
4 & 74 & $\mathrm{M}$ & $\mathrm{Si}$ & Crónica & VATS + toracotomía & IV & 5 meses \\
\hline
\end{tabular}

*VATS: Cirugía torácica asistida por video 
elevada. En la tomografía computarizada (TC) de tórax se observa colección pleural posterior derecha. Se decide realizar nueva decorticación por VATS, con hallazgo intraoperatorio de empiema en fase de organización sin adecuada reexpansión pulmonar, por lo que se practica toracostomía abierta con resección parcial del octavo arco costal. En el postoperatorio se maneja con curación diaria con lavado de cavidad y empaquetamiento con compresas estériles impregnadas con solución salina, con evolución clínica satisfactoria y sin recurrencia del empiema durante el seguimiento.

\section{Caso clínico 2}

Paciente femenina de 60 años de edad, con antecedente de hipertensión arterial, hipotiroidismo en suplencia hormonal y trauma cerrado de tórax secundario a accidente automovilístico ocurrido 4 meses previo al ingreso, donde presentó fracturas costales múltiples y hemotórax coagulado, que requirió drenaje y decorticación por VATS. Ingresa por cuadro de secreción purulenta por heridas en tórax de un mes de evolución aproximadamente. La TC de tórax simple mostró colección pleural izquierda de $82 \mathrm{~mm}$ con atelectasia casi completa del lóbulo pulmonar inferior izquierdo. Se decidió llevar a decorticación pulmonar por VATS, pero cuando se ingresa a cavidad pleural se identifica fibrosis pleural severa, que no permite continuar procedimiento por este abordaje. Debido a la morbilidad, trauma con fracturas costales e intervenciones previas en tórax, se decide realizar toracostomía abierta. En el postoperatorio se maneja con curación diaria con lavado de cavidad y empaquetamiento con compresas estériles impregnada con solución salina, con evolución clínica satisfactoria y sin recurrencia durante el seguimiento.

\section{Caso clínico 3}

Paciente masculino de 51 años de edad, con hospitalización el mes previo al ingreso por obstrucción intestinal, que requirió resección intestinal y anastomosis, con manejo posterior en la unidad de cuidado intensivo, donde desarrolla fungemia por cándida y tromboembolismo pulmonar agudo.
Evolución clínica lenta hacia la mejoría, por lo que se decide dar egreso. Reingresó a los 7 días por cuadro clínico consistente en disnea y expectoración amarilla, asociado a respuesta inflamatoria sistémica. Se realizó radiografía de tórax que mostró derrame pleural masivo y hemograma con anemia severa, por lo que se sospechó hemotórax y se decidió someter a toracostomía cerrada, con de salida de $500 \mathrm{cc}$ de pus.

Se tomó nuevo TC de tórax, en el que se evidenció colección posterobasal derecha, el cual no estaba en contacto con el tubo de tórax, por lo que se planteó la realización de decorticación pulmonar por VATS. Se llevó al procedimiento quirúrgico encontrando una colección pleural superior derecha de $200 \mathrm{cc}$, cavitada, con múltiples adherencias pleuropulmonares firmes, severo engrosamiento de la pleura parietal y visceral, con atrapamiento pulmonar. Se decide proceder a toracostomía abierta con resección parcial del quinto arco costal, por las comorbilidades, desnutrición y hallazgos intraoperatorios. En el postoperatorio se manejó de igual forma, con evolución clínica satisfactoria y sin recurrencia durante el seguimiento.

\section{Caso clínico 4}

Paciente de 75 años de edad con antecedente de diabetes mellitus tipo 2, hipertensión arterial y enfermedad pulmonar obstructiva crónica, que ingresa al servicio de urgencias por cuadro clínico de disnea, deterioro de la clase funcional y dolor pleurítico. Se realizaron estudios encontrándose derrame pleural con liquido compatible con empiema, por lo que se decidió llevar a cirugía para decorticación por toracoscopia. Durante el procedimiento se drenaron 2900 cc de pus, y fue necesario convertir a procedimiento abierto (toracotomía posterolateral) por sangrado, logrando su control por esta vía. A los 10 días posoperatorios se decide egreso hospitalario ante la adecuada evolución clínica.

El paciente reingresó al servicio de urgencias a los 15 días por fiebre y dificultad respiratoria y se diagnosticó un choque séptico, por lo que se realizó una TC de tórax que mostró una colección compleja en el hemitórax izquierdo, ocupando casi 
toda la extensión del hemitórax, de paredes gruesas (figura 1). Ante los hallazgos de empiema recurrente y en fase organizada, se plantea un nuevo procedimiento quirúrgico, no obstante, siendo un paciente que se encontraba en condición de fragilidad, se decide cambiar de estrategia terapéutica, realizando una toracostomía abierta con resección parcial del séptimo y octavo arcos costales.

Se decidió en este paciente manejar la toracostomía abierta con terapia de presión negativa, (VAC, vacuum-assisted closure), intratorácica (figura 2), y fue necesario cambio del VAC en 5 oportunidades, las cuales se realizaron en promedio cada quinto día. Los tres primeros cambios se realizaron con el paciente hospitalizado y para los otros dos, el paciente fue manejado en su domicilio con la terapia VAC, asistiendo al hospital para cambio del mismo. El paciente durante su evolución posoperatoria presenta una mejoría no-
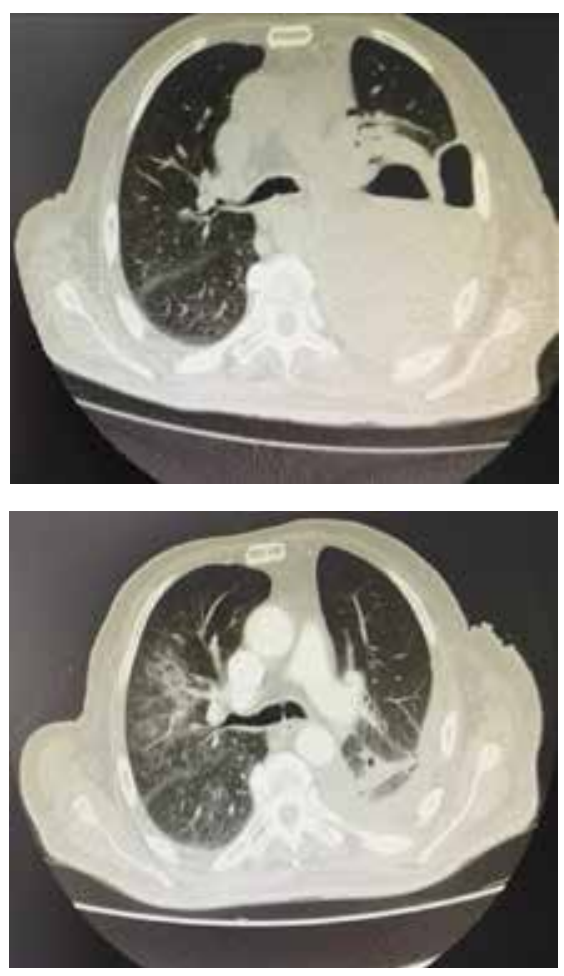

Figura 1. Colección hidroaérea compleja, de paredes gruesas, ocupando casi toda la extensión del hemitórax izquierdo, con unos diámetros mayores de $28 \times 14 \times 10 \mathrm{~cm}$ (LXAPxT). table, con modulación de la respuesta inflamatoria sistémica y mejoría de la dificultad respiratoria. Al finalizar la terapia el paciente presenta obliteración completa del espacio pleural y cierre parcial de la toracostomía (figura 3), con posterior manejo complementario por clínica de heridas.

\section{Discusión}

Una opción de tratamiento para la tercera fase del empiema es la toracostomía abierta, inicialmente descrita por Eloesser, en donde se reseca parte de 1 a 3 costillas, en la zona más próxima a la colección ${ }^{10}$. Suele ser un procedimiento mutilante, en

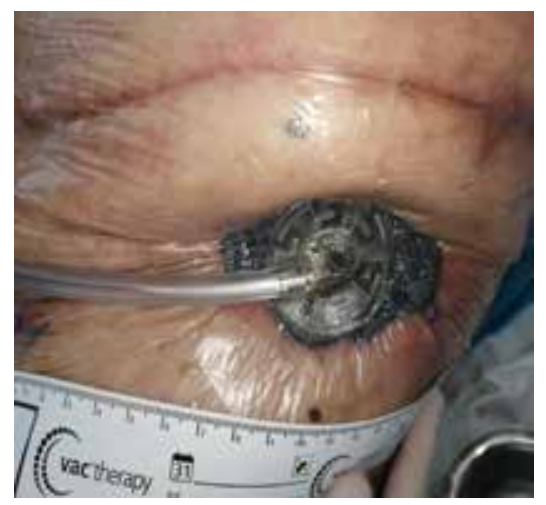

Figura 2. En la parte superior se observa la cicatriz de la toracotomía posterolateral previa. En la parte inferior se observa la toracostomía abierta en manejo con VAC.

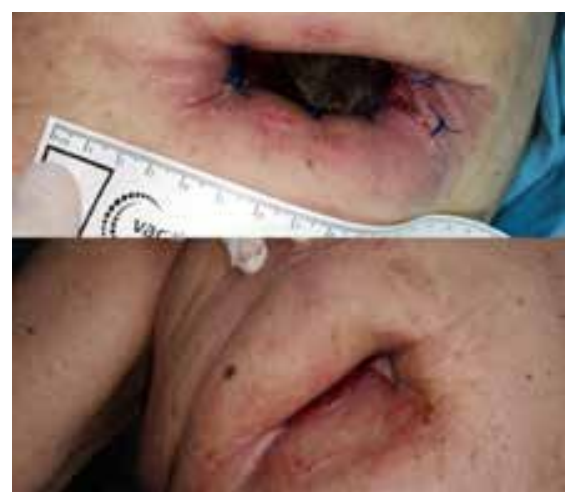

Figura 3. En la vista superior se aprecia la toracostomía abierta con espacio pleural amplio y membranas fibrinopurulentas; en la vista inferior, la toracostomía abierta después del tercer cambio de VAC, donde se observa cierre progresivo y tejido de granulación. 
el que se requiere de varios meses para su cierre, o inclusive de reconstrucción quirúrgica, y puede haber dolor crónico. En nuestro medio viene en desuso, debido al uso de antibióticos asociado a otras opciones quirúrgicas, como la toracostomía cerrada y la decorticación por VATS, sin embargo, en pacientes seleccionados la toracostomía abierta puede llegar a ser la mejor opción para el control del foco infeccioso ${ }^{11-13}$.

Actualmente la indicación más frecuente para toracostomía abierta es el empiema posterior a neumonectomía ${ }^{11,12}$. En nuestra serie este no era el caso: se trató de pacientes con múltiples comorbilidades, ASA III-IV y en estado de fragilidad, por lo que se eligió este procedimiento. Adicionalmente, cabe destacar que el empiema se encontraba en fase de organización y en tres de los cuatro casos era recurrente.

La terapia VAC intratorácica para el manejo del empiema fue descrita por primera vez en el 2006 ${ }^{14} \mathrm{y}$ desde esta primera descripción se han realizado múltiples estudios donde se demuestra el beneficio de la terapia de presión negativa debido al más rápido control de la sepsis y control local de la cavidad pleural, además de mejor función pulmonar por favorecer la reexpansión, sin estar contraindicada en caso de fistulas ${ }^{4,7}$.

Otra opción descrita para el manejo del empiema pleural es la colocación de VAC dentro de la cavidad pleural, sin la necesidad de realizar una toracostomía abierta, la que se denomina terapia VAC mínimamente invasiva ${ }^{15}$

En cuanto al cierre de la toracostomía abierta, tres de los pacientes tuvieron un cierre por segunda intención en manejo con clínica de heridas únicamente, entre los que está incluido el paciente que se manejó con terapia VAC, sin requerir un procedimiento quirúrgico adicional en ellos. El paciente restante no continuó el seguimiento en nuestro servicio.

Definitivamente, en estos cuatro casos que presentamos, dadas las condiciones clínicas de los pacientes, la toracostomía abierta fue la mejor estrategia terapéutica, de acuerdo con las recomendaciones de la Asociación Americana de Cirugía de Tórax, que establece la práctica de toracostomía abierta para el manejo del empiema crónico en pacientes incapaces a tolerar una decorticación, y sugiere al VAC como una opción razonable para el manejo de la herida ${ }^{12}$.

Para concluir, es importante destacar que la toracostomía abierta es el tratamiento de elección en casos seleccionados, donde la toracostomía cerrada y la decorticación no pueden realizarse o han fracaso. A su vez, se puede asociar a un VAC para obliterar el espacio pleural, ofreciendo una mejor calidad de vida para el paciente y una baja tasa de reinfección. No obstante, se debe tener en cuenta que este procedimiento debe ser realizado por alguien capacitado, con la experiencia suficiente, debido a las posibles complicaciones.

Por ahora, es necesario realizar más estudios para lograr un mayor nivel de evidencia de estas recomendaciones.

\section{Cumplimiento de normas éticas}

Consentimiento informado. Se cuenta con el consentimiento informado por parte de los pacientes para la publicación de cada caso. Además, se obtuvo el aval de la Dirección científica de Méderi.

Conflicto de intereses. Los autores refirieron que no hay conflicto de intereses.

Financiación. Recursos propios de los autores.

Contribución de los autores. Diseño, interpretación de datos y redacción: Camilo Ramírez-Giraldo. Búsqueda de datos y revisión crítica: Fiamma Vásquez. Interpretación de datos y revisión crítica: Mauricio Peláez Arango.

Todos los autores participaron en la aprobación final del manuscrito.

\section{Referencias}

1. Taylor MD, Kozower BD. Surgical spectrum in the management of empyemas. Thorac Surg Clin. 2012;22:43140. https://doi.org/10.1016/j.thorsurg.2012.04.007

2. Enfield KB, Sifri CD. Aspiration, empyema, lung abscesses, and anaerobic infections. In: Grippi MA, editor Fishman's Pulmonary Diseases and Disorders. Fifth Edit. New York: McGraw-Hill; 2015.

3. Semenkovich TR, Olsen MA, Puri V, Meyers BF, Kozower BD. Current state of empyema management. Ann Thorac Surg. 2018;105:1589-96.

https://doi.org/10.1016/j.athoracsur.2018.02.027 
4. Sziklavari Z, Grosser C, Neu R, Schemm R, Kortner A, Szöke T, Hofmann HS. Complex pleural empyema can be safely treated with vacuum-assisted closure. J Cardiothorac Surg. 2011;6:130. https://doi.org/10.1186/1749-8090-6-130

5. Salguero J, Cardemil G, Molina JC, Lembach H, Fernández J. Empiema pleural: Etiología, tratamiento y complicaciones. Rev Chil Cir. 2009;61:223-8. http: / / dx.doi.org/10.4067/S0718-40262009000300003

6. Massera F, Robustellini M, Della Pona C, Rossi G, Rizzi A, Rocco G. Open window thoracostomy for pleural empyema complicating partial lung resection. Ann Thorac Surg. 2009;87:869-74.

http://doi.org/10.1016/j.athoracsur.2008.12.003

7. Palmen M, van Breugel HNAM, Geskes GG, van Belle A, Swennen JMH, Drijkoningen AHM, et al. Open window thoracostomy treatment of empyema is accelerated by vacuum-assisted closure. Ann Thorac Surg. 2009;88:1131-6. http://doi.org/10.1016/j.athoracsur.2009.06.030

8. Sziklavari Z, Ried M, Zeman F, Grosser C, Szöke T, Neu $\mathrm{R}$, et al. Short-term and long-term outcomes of intrathoracic vacuum therapy of empyema in debilitated patients. J Cardiothorac Surg. 2016;11:1-8. http://doi.org/10.1186/s13019-016-0543-7

9. Maeda S, Sado T, Sakurada A, Okada Y, Kondo T. Successful closure of an open-window thoracostomy wound by negative-pressure wound therapy: report of a case. Surg Today. 2012;42:295-8.

http://doi.org/10.1007/s00595-011-0008-5

10. Denlinger CE. Eloesser flap thoracostomy window. Oper Tech Thorac Cardiovasc Surg. 2010;15:61-9. http://doi.org/10.1053/j.optechstcvs.2010.03.003

11. Reyes KG, Mason DP, Murthy SC, Su JW, Rice TW. Open window thoracostomy: Modern update of an ancient operation. Thorac Cardiov Surg. 2010;58:220-4. http://doi.org/10.1055/s-0029-1240972

12. Shen KR, Bribriesco A, Crabtree T, Denlinger C, Eby J, Eiken $\mathrm{P}$, et al. The American Association for Thoracic Surgery consensus guidelines for the management of empyema. J Thorac Cardiovasc Surg. 2017;153:e12946. http://doi.org/10.1016/j.jtcvs.2017.01.030

13. Tapias L, Tapias-Vargas LF, Tapias-Vargas L. Complicaciones de los tubos de tórax. Rev Colomb Cir. 2009;24:46-55.

14. Ditterich D, Rexer M, Rupprecht H. Vacuum assisted closure in the treatment of pleural empyema - First experiences with intrathoracal application. Zentralbl Chir. 2006;131(Suppl. 1):S133-S138. http://doi.org/10.1055/s-2006-921499

15. Hofmann HS, Neu R, Potzger T, Schemm R, Grosser C, Szöke T, Sziklavari Z. Minimally invasive vacuum-assisted closure therapy with instillation (mini-vac-instill) for pleural empyema. Surg Innov. 2015 Jun;22:235-9. https://doi.org/10.1177/1553350614540811 